\title{
Mesenchymal Stem Cell-Encapsulated Collagen Microspheres for Bone Tissue Engineering
}

\author{
Barbara Pui Chan, Ph.D.,,2 Ting Yan Hui, M.Phil.,, ${ }^{1,2}$ Mei Yi Wong, B.Eng.,., \\ Kevin Hak Kong Yip, B.D.S., Ph.D., ${ }^{3}$ and Godfrey Chi Fung Chan, M.D. ${ }^{4}$
}

There is a demonstrated clinical need for alternatives of autologous fresh bone graft with excellent biological performance in osteoconductivity, osteoinductivity, and osteogenicity. We previously developed a collagen microencapsulation technology entrapping bone marrow-derived mesenchymal stem cells (MSCs) in a biomimetic collagen fiber meshwork and produced injectable collagen-MSC microspheres. In this study, we hypothesize that injectable microspheres with osteoconductivity, osteogenicity, and osteoinductivity can be fabricated by differentiating the encapsulated MSCs, from either human or mouse sources, toward osteogenic lineages in these three-dimensional microspheres. The osteogenicity, osteoconductivity, and osteoinductivity of the microspheres were evaluated in vitro. Osteogenic markers of the differentiating MSCs including alkaline phosphatase and calcium deposition showed positive staining. Osteoconductivity of the collagen meshwork in the microsphere was demonstrated by the presence of calcium phosphate deposits among the collagen fibers and by the significantly increased calcium content extracted from the microspheres. Moreover, osteoinductivity of the MSC-encapsulated microspheres was demonstrated by the ability to induce osteogenic differentiation of undifferentiated MSCs in both contact and noncontact coculture. This study contributes toward the future development of injectable alternatives for fresh bone grafts using autologous MSCs.

\section{Introduction}

$\mathbf{T}$ HERE IS A DEMONSTRATED CLINICAL NEED for alternatives of fresh bone grafts in treating bone disorders. ${ }^{1-3}$ Autologous bone grafts are the gold standard for bone repair and regeneration. ${ }^{4}$ This is mainly because of the absence of immunogenicity and the presence of three favorable characteristics for bone regeneration-namely, osteoinductivity, osteoconductivity, and osteogenicity. ${ }^{1,3,5}$ Nevertheless, invasive nature of the harvesting procedure, donor-site morbidities, and limited availability are the main drawbacks of autologous bone grafting irrespective of the excellent performance of this approach. Allogenic or xenogenic bone grafts do not have the donor-site morbidity and the availability issues but present the immunogenicity problem and have compromised the biological performance as compared to autografts. ${ }^{6,7}$

Upon Urist's initial report on the osteoinductivity of demineralized and freeze-dried allogenic grafts, ${ }^{8}$ the active compounds responsible for the osteoinductivity, namely, bone morphogenic proteins (BMPs), have been identified and researched extensively, ${ }^{9,10}$ and the demineralized bone matrix possessing osteoinductivity has been developed into various formulations. ${ }^{2,11}$ Nevertheless, multiple injections of BMPs are needed, and better carriers able to control their release are therefore needed to make them an affordable option. For the demineralized bone matrix, mechanically stable carriers and augmentation formulations are needed to improve their handling capability. ${ }^{2}$

Osteoconductivity of bone grafts is the ability to provide three-dimensional (3D) porous structures facilitating cell and nutrient infiltration for bone growth. ${ }^{1,3}$ Many biomaterials with osteoconductivity have been developed as bone graft substitutes to provide readily available scaffolds facilitating bone ingrowth. ${ }^{12}$ Examples include calcium phosphatebased materials, such as insoluble hydroxyapatites and soluble calcium sulfates, ${ }^{1,12,13}$ and collagen type I. ${ }^{14}$ However, osteoconductive materials alone are of limited applications as they require osteoinductive supplements and osteogenic cell sources for bone regeneration.

\footnotetext{
${ }^{1}$ Medical Engineering Program, Department of Mechanical Engineering, The University of Hong Kong, Hong Kong Special Administrative Region, China.

2Department of Mechanical Engineering, The University of Hong Kong, Hong Kong Special Administrative Region, China.

${ }^{3}$ Restorative Dentistry, School of Medicine and Dentistry-A2.237, James Cook University, Cairns Qld, Australia.

${ }^{4}$ Department of Paediatrics and Adolescent Medicine, Li Ka Shing Faculty of Medicine, The University of Hong Kong, Hong Kong Special Administrative Region, China.
} 
Osteogenic activity refers to the provision of bone-forming cells such as osteoblastic cells from fresh bone grafts or bone marrow. ${ }^{1,15}$ Bone marrow-derived mesenchymal stem cells (MSCs) are multipotent cells able to differentiate into multiple cell types, including osteogenic lineages ${ }^{16}$ and therefore can be used as an osteogenic cell source. Important roles of bone marrow-derived MSCs treating bone disorders have been reported. ${ }^{17-19}$ Moreover, the possibility of using autologous bone marrow-derived MSCs as the osteogenic cell source assures better biological performance for bone regeneration.

We previously developed a novel collagen microencapsulation technology, ${ }^{20}$ which entraps bone marrow MSCs in a self-assembled biomimetic collagen matrix consisting of collagen fiber meshwork. These stable and injectable microspheres provide a physiologically relevant microenvironment to support MSC viability, proliferation, migration, and differentiation have been produced for cell delivery and tissue engineering. ${ }^{21}$ Recently, we successfully induced the encapsulated bone marrow MSCs into chondrogenic lineage, which remodels the fibrous meshwork with cartilage-specific type II collagen and aggrecan matrix, producing injectable cartilage tissue micromasses. ${ }^{22}$ In this study, we hypothesize that injectable microspheres with osteogenic, osteoconductive, and osteoinductive activities can be fabricated by differentiating human MSCs (hMSCs) or mouse MSCs (mMSCs) encapsulated in these 3D microspheres toward osteogenic lineages. Osteogenic markers such as alkaline phosphatase (ALP) and calcium deposition of the differentiating MSCs will be evaluated by cytochemical staining. Osteoconductivity of the collagen microsphere will be evaluated by microstructural analysis using scanning electron microscopy (SEM) and energy dispersive X-ray (EDX) and quantitative calcium content analysis. Osteoinductivity of the osteogenic differentiated MSC-encapsulated microspheres will be evaluated by contact and noncontact coculture with undifferentiated MSCs.

\section{Materials and Methods}

\section{Isolation and culture of hMSC}

Human bone marrow aspiration and isolation and culture of hMSCs were conducted as previously described. ${ }^{23}$ In brief, bone marrow aspirates were collected from three healthy donors, in accordance with the protocol approved by the Combined Clinical Ethics Committee of the University of Hong Kong and Hong Kong West Cluster Hospitals of Hospital Authority. Mononuclear cells were isolated by a FicollHypaque (Amersham Biosciences, Uppsala, Sweden) density gradient and cultured in growth medium consisting of Dulbecco's modified Eagle's medium-low glucose (DMEM-LG), $10 \%$ fetal bovine serum (FBS), $100 \mathrm{U} / \mathrm{mL}$ penicillin, $100 \mu \mathrm{g} / \mathrm{mL}$ streptomycin, and $2 \mathrm{mM}$ L-glutamine. Cultures were maintained at $37^{\circ} \mathrm{C}$ in a humidified atmosphere with $5 \% \mathrm{CO}_{2}$. MSCs were selected by adherence after $24 \mathrm{~h}$ and maintained in growth medium with replacement every 3-4 days. hMSCs in P1 to P5 were used. The immunophenotype, self-renewal capacity, and the multiple differentiating potential of the isolated and cultured hMSCs have been verified previously. ${ }^{21,23}$

\section{Isolation and culture of $\mathrm{mMSC}$}

Animal experimentation was conducted with appropriate ethics approval according to institutional regulations. In brief, bone marrow was flushed out from the femurs and tibias of green fluorescent protein (GFP)-mouse (ICR) using a 30-gauge needle and collected into a $50 \mathrm{~mL}$ centrifuge tube through a cell strainer. Cell suspensions obtained were centrifuged at $400 \mathrm{~g}$ for $10 \mathrm{~min}$ before seeding in T75 culture flasks. DMEM-LG (Gibco, Carlsbad, CA) supplemented with sodium bicarbonate, $10 \mathrm{mM} \mathrm{HEPESs}$, and 10\% FBS was used to culture the cells. Growth medium was changed after 3 days of culture and replenished every 3-4 days thereafter. mMSCs were subcultured, and cells in P1 were used.

\section{Microencapsulation of MSCs in collagen microspheres}

Collagen-MSC microspheres were prepared as previously described. ${ }^{21}$ In brief, MSC suspensions at a final concentration of $5 \times 10^{5}$ cells $/ \mathrm{mL}$ were mixed with neutralized rat tail type I collagen solution (BD Biosciences, Bedford, MA) at a final concentration of $2 \mathrm{mg} / \mathrm{mL}$, unless otherwise specified, in an ice-bath. Droplets of $2.5 \mu \mathrm{L}$ were pipetted into $35-\mathrm{mm}$ diameter culture dishes covered with ultraviolet-irradiated parafilm to prevent adhesion of the microsphere to the substratum. The collagen-MSC mixtures underwent gelation when incubated at $37^{\circ} \mathrm{C}$ in a humidified atmosphere with $5 \%$ $\mathrm{CO}_{2}$ for $30-45 \mathrm{~min}$.

\section{Osteogenic differentiation of MSC in collagen microspheres}

The microspheres were cultured in DMEM-LG supplemented with $10 \%$ FBS, $100 \mathrm{nM}$ dexamethasone, $50 \mu \mathrm{M}$ ascorbic acid 2-phosphate, and $10 \mathrm{mM} \beta$-glycerophosphate as the osteogenic differentiation induction medium. To compare the osteogenic differentiation efficiency between mMSC and hMSC, in each species, 300 collagen-MSC microspheres were seeded in a 100-mm-diameter culture dish, in triplicates, and were maintained in the differentiation induction medium for 14 and 19 days with regular medium replenishment. In a separate experiment, 100 collagen-mMSC microspheres with final collagen concentration at $3 \mathrm{mg} / \mathrm{mL}$ were produced and cultured in differentiation medium for 7, 14, and 21 days, in triplicates, to trace the temporal change in calcium content over time during differentiation.

\section{Histological and immunohistochemical analysis for osteogenic markers}

Samples were fixed with $4 \%$ phosphate buffered salinebuffered paraformaldehyde and cut into $10 \mu \mathrm{m}$ paraffin and frozen sections. The sections were stained with hematoxylin and eosin (H\&E) for histological analyses. ALP is an early marker for osteogenic differentiation. During ALP staining, magenta solution, the initiator, and TAC buffer (Sigma, Dorset, United Kingdom) were mixed at a ratio of 1:1:50 in darkness. Droplets of the substrate reagent were added on the sections and incubated for 10-60 min. The slides were then rinsed in distilled water, dehydrated, and mounted. von Kossa staining was used to reveal the presence of calcium deposits, a marker for advanced stage of osteogenic differentiation. In brief, sections were incubated with $1 \%$ silver nitrate solution (Sigma) in darkness for $30 \mathrm{~min}$ and were irradiated under ultraviolet light for $1 \mathrm{~h}$. Unreacted silver was removed by incubating with $2 \%$ sodium thiosulfate for $5 \mathrm{~min}$. Nuclear fast red was used as a counter stain. 


\section{SEM and EDX for microstructural analysis}

To examine the microstructure of the collagen-hMSC microspheres, samples were processed for SEM analysis. The microspheres were rinsed with phosphate-buffered saline and fixed in $2.5 \%$ glutaraldehyde for $2 \mathrm{~h}$ at $4{ }^{\circ} \mathrm{C}$. After dehydration through a graded series of ethanol, the microspheres were critical point dried and fractured to expose their cross sections. The microspheres were examined with SEM coupled with EDX spectrometry (LEO 1530; LEO Electron Microscopy, Cambridge, UK) for microstructural analysis and for detection of calcium and phosphorus and their relative distribution in the samples. The quantities of these elements were measured, and the calcium to phosphorus ratios calculated.

\section{Quantification of calcium content after extraction}

Calcium deposits in the collagen meshwork of the microspheres were extracted with $1 \%$ trichloroacetic acid for $24 \mathrm{~h}$ before quantification by calcium assay kit (Bioassay Systems, Hayward, CA, Cat\#: DICA-500). In brief, equal volumes of Reagent $A$ and Reagent $B$ were combined and equilibrated to room temperature before use. Standard solutions of $\mathrm{Ca}^{2+}$ $(12.5-200 \mu \mathrm{g} / \mathrm{mL})$ were prepared by serial dilution in distilled water. An aliquot of $5 \mu \mathrm{L}$ standards or samples was transferred into wells of a clear-bottom 96-well plate, and $200 \mu \mathrm{L}$ of working reagent was added. The mixture was incubated at room temperature for $3 \mathrm{~min}$ before measuring the absorbance at $612 \mathrm{~nm}$. The amounts of $\mathrm{Ca}^{2+}$ present in the samples were determined by calibrating against the linear region of the standard curve.

\section{In vitro osteoinductivity assay}

Osteoinductivity refers to the ability to induce differentiation of osteogenic cell sources such as MSCs into osteogenic lineage and therefore induce bone formation. ${ }^{24}$ The osteoinductive activity of the differentiated microspheres was evaluated by contact and noncontact coculture with undifferentiated MSCs. In brief, undifferentiated MSCs were seeded in the lower chamber of a 24-well Transwell culture plate (BD Biosciences) at a density of $1.5 \times 10^{4}$ cells/well for 3-4 days. Five hundred osteogenically differentiated microspheres with GFP-mMSCs were placed in the upper chamber of the transwell for noncontact coculture in duplicates. Growth medium was supplemented to both the upper and the lower chambers with regular replenishment every 3 days for 14 days. In separate 24-well culture dishes (BD Biosciences), undifferentiated MSCs at the same seeding densities were cultured. Same amount of osteogenically differentiated microspheres were plated on the monolayer cultures of the undifferentiated MSCs for contact coculture in duplicates. Normal growth medium was supplemented and exchanged regularly. At the end of incubation period of 14 days, ALP staining for osteogenically differentiated MSCs and von Kossa staining for calcium deposits were used to evaluate whether the undifferentiated MSCs were induced to differentiate toward the osteogenic lineages.

\section{Statistical analysis}

Data were presented as means with standard deviations. The normality assumption was verified before conducting parametric tests. Two-way analysis of variance with appropriate post hoc tests were used to compare the differences in dry weight and calcium content in different groups and among different time points. One-way analysis of variance with linear trend analysis was used to compare the dry weight and calcium content at different time points during osteogenic differentiation. SPSS (Chicago, IL) 16.0 was used to execute the analyses, and the significance level was set at $p<0.05$.

\section{Results}

\section{Morphological changes of collagen-MSC microspheres upon osteogenic differentiation}

Figure 1 shows the morphological changes of the collagen microspheres encapsulating hMSC and mMSC during osteogenic differentiation. In general, microspheres cultured in normal growth medium contracted continuously over time, whereas microspheres exposed to osteogenic differentiation medium maintained their sizes at around $250 \mu \mathrm{m}$ in diameter soon after the initial contraction. In collagen-mMSC microspheres, calcium deposits in the form of tiny dark granules (arrows) were found throughout the microspheres on day 5 under the phase contrast microscopy (right panel, Fig. 1). Calcium deposition continued and the whole microsphere becomes dark and opaque as soon as day 8 , and the microspheres further darkened over time. In microspheres with hMSCs, dark patches (arrows) appeared later than those with mMSC in the second week (day 13) upon differentiation (left panel, Fig. 1). The microsphere becomes darker in the third week (day 21), but some regions in the microsphere were still transparent, leading to inhomogeneous darkness. Control microspheres without osteogenic differentiation for both hMSC and mMSC were transparent without obvious calcium deposits throughout the culture period.

\section{Histological and histochemical analysis of collagen-MSC microspheres}

Figure 2 shows the H\&E staining and the von Kossa staining of collagen-MSC microspheres for both mMSC and hMSC. H\&E staining showed the cell and microsphere morphology. In brief, microspheres with mMSC showed hypocellularity with basophilic matrix after osteogenic differentiation (Fig. 2B) in comparison with eosinophilic matrix in control microspheres (Fig. 2A). von Kossa staining showed the calcium deposition within the microspheres. In brief, the microsphere was heavily stained brown, suggesting calcium phosphate deposits (Fig. 2D) in contrast with the negative staining in control microspheres (Fig. 2C). Microspheres with hMSCs with (Fig. 2F, H) and without differentiation were shown (Fig. 2E, G). Some grayish/brownish deposits in von Kossa staining were found (Fig. $2 \mathrm{H}$ ) although the signal was much lower than that of mMSC (Fig. 2D).

\section{SEM analysis of collagen-MSC microspheres after osteogenic differentiation}

During osteogenic differentiation, calcium granules deposited within the fiber meshwork. Figure 3 shows the SEM images of collagen-MSC microspheres. The microstructure of the undifferentiated microsphere is mainly meshwork of nanosized collagen fibers (Fig. 3A), confirming our previous 
hMSC

\section{Osteogenic differentiation}
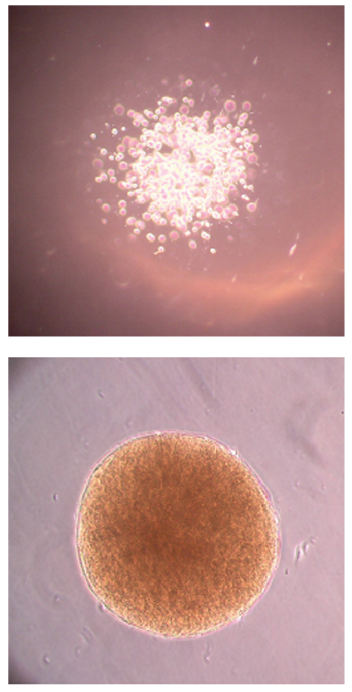

Day 3

\section{Day 0}

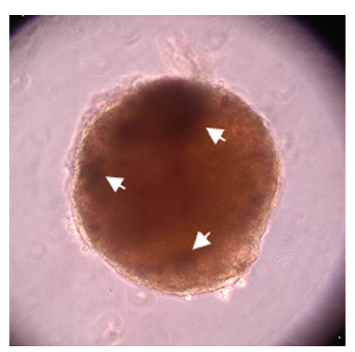

Day 13

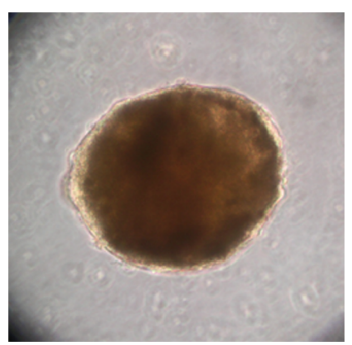

Control
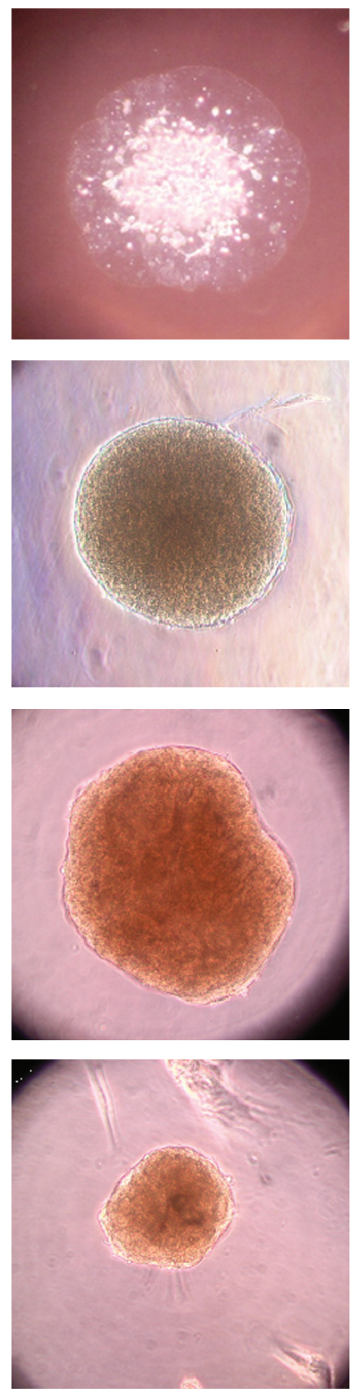

Day 0
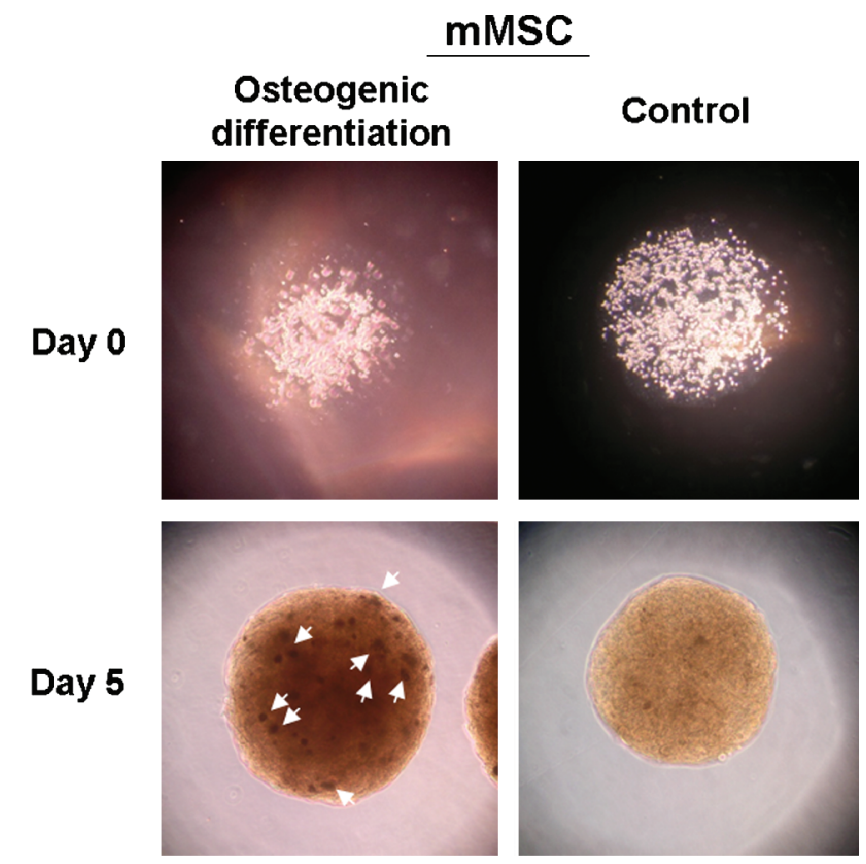

Day 8
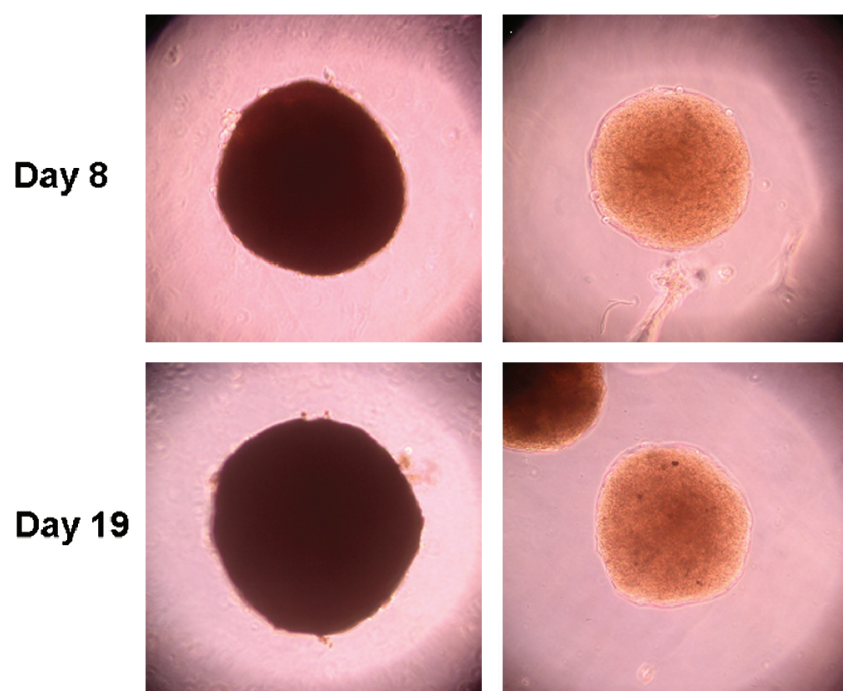

FIG. 1. Phase contrast images of collagen microspheres encapsulating mouse mesenchymal stem cell (mMSC) and human MSC (hMSC) at different time points during osteogenic differentiation. Left panel: morphology of hMSC-encapsulated collagen microspheres in osteogenically differentiation and control groups on days $0,3,13$, and 21 postencapsulation. Right panel: morphology of mMSC-encapsulated collagen microspheres in osteogenic differentiation and control groups on days 0 , 5, 8, and 19 postencapsulation. Magnifications: day 0, $4 \times$; all others, $10 \times$ (arrows indicate dark calcium deposits in the shape of granules or patches). Color images available online at www.liebertonline.com/ten.

results. $^{21}$ Upon osteogenic differentiation, there were numerous granules and flakes ${ }^{*}$ ) deposited on the nanofibrous meshwork (arrows) of the microsphere with mMSCs (Fig. 3B, C), and the fiber meshwork was less visible than before as the fibers were covered by the deposits. Under magnified views, these granules and flakes were around $200 \mathrm{~nm}$ in diameter and intercalated with the collagen fibers (arrows) (Fig. 3C). In microspheres with hMSCs, there were less calcium deposits and the granules were of much smaller size (Fig. 3D, E). After calcium extraction, the microstructure of the microsphere resembled the undifferentiated microspheres with collagen fiber meshwork only and without calcium granules or flakes (Fig. 3F).

\section{EDX analysis of collagen-MSC microspheres after osteogenic differentiation}

Figure 4 shows the EDX analysis for calcium and phosphorus elements in the microspheres. The spectrum of different elements present in the microspheres during osteogenic differentiation showed much higher peak heights for calcium and phosphorus (Fig. 4A) than those in the control microspheres (Fig. 4D). The result was further confirmed by the higher intensity of positive signals in the mappings of the EDX analysis for calcium and phosphorus in differentiated samples (Fig. 4B, C) than control samples (Fig. 4E, F). Moreover, the signals for both calcium and phosphorus in 


\section{H\&E staining}

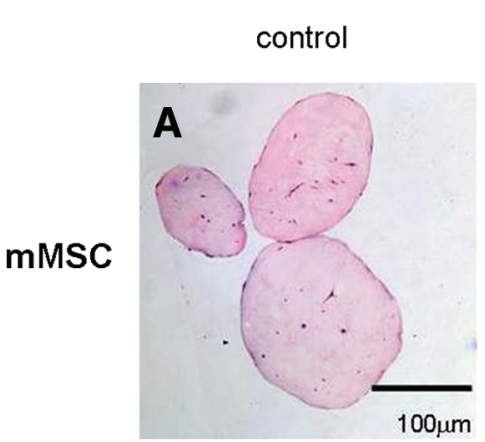

hMSC

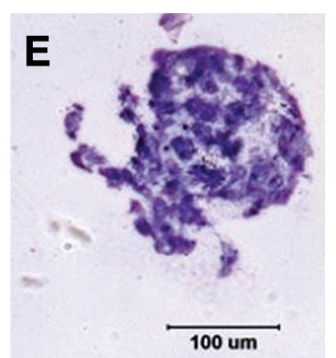

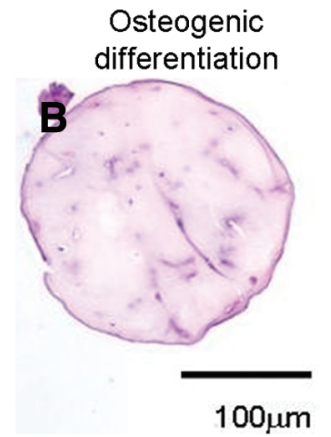

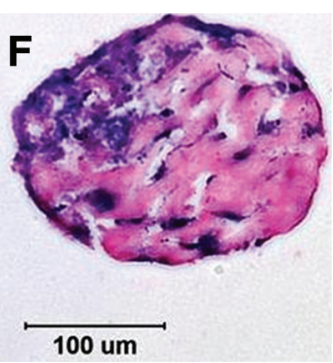

von Kossa staining

control

C

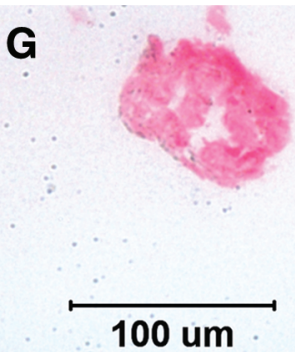

Osteogenic differentiation
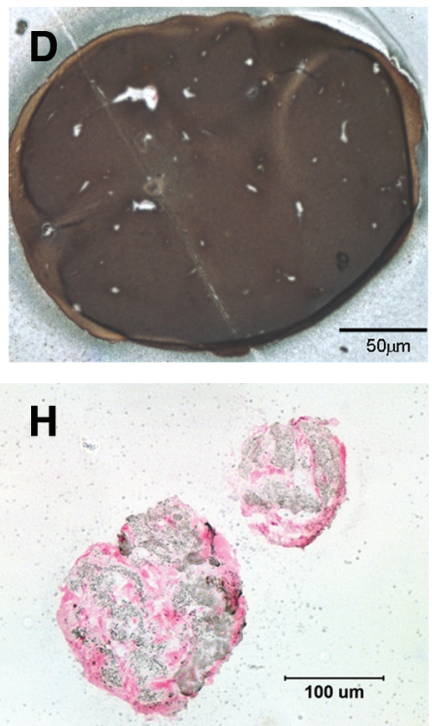

FIG. 2. H\&E and von Kossa staining of mMSC- and hMSC-collagen microspheres in osteogenic differentiation and control groups at 21 days post-differentiation. (A-D): mMSC; (E-H): hMSC; (A, B, E, F): H\&E staining; (C, D, G, H): von Kossa staining; (A, E, C, G): Control group; (B, F, D, H): Osteogenic differentiation group. Color images available online at www .liebertonline.com/ten.

the samples were homogeneously distributed throughout the area inspected. The average calcium and phosphorus content of the samples and the controls were shown in the lower panel of Figure 4. Osteogenically differentiated microspheres contained a higher weight percentage in both elements than the controls. Nevertheless, the weight percentage results are not fully quantitative as the composition was affected by many factors, including the sample thickness and roughness, which are difficult to control in the microsphere configuration. As a result, quantitative analysis for calcium content
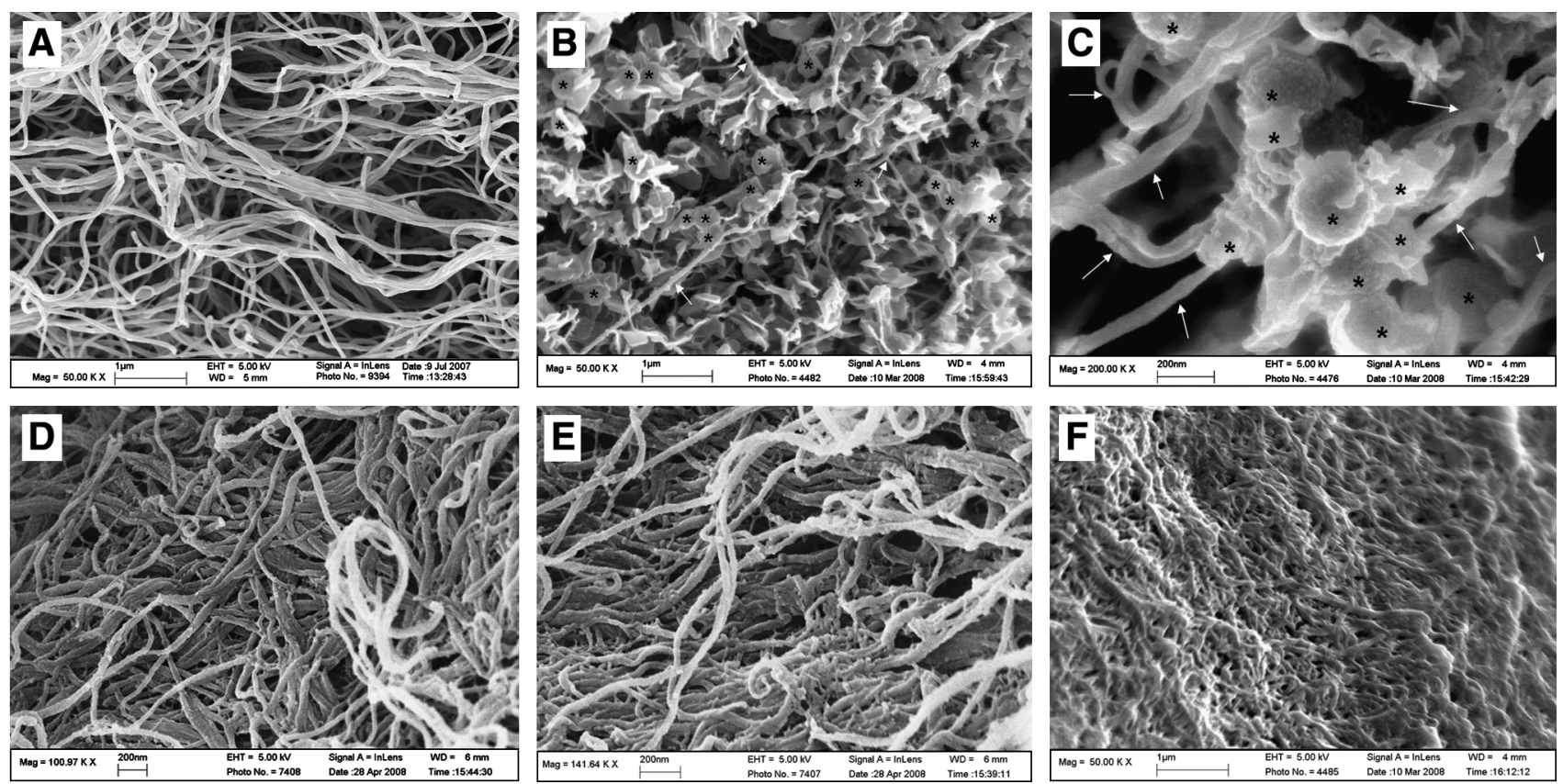

FIG. 3. Scanning electron microscopy of collagen-MSC microspheres before and after calcium extraction. Scanning electron microscopy pictures revealed the microstructure of collagen-MSC microspheres before osteogenic differentiation (A) and 21 days after osteogenic differentiation (B, C: mMSC; D, E: hMSC) as well as after calcium extraction (F). Magnification: $50 \mathrm{k} \times$ $(\mathbf{A}, \mathbf{B}, \mathbf{F}) ; 200 \mathrm{k} \times(\mathbf{C}) ; 100 \mathrm{k} \times(\mathbf{D}) ; 142 \mathrm{k} \times(\mathbf{E})$. Asterisk indicates calcium deposits in the shape of flakes or granules; arrows indicate collagen fibers. 


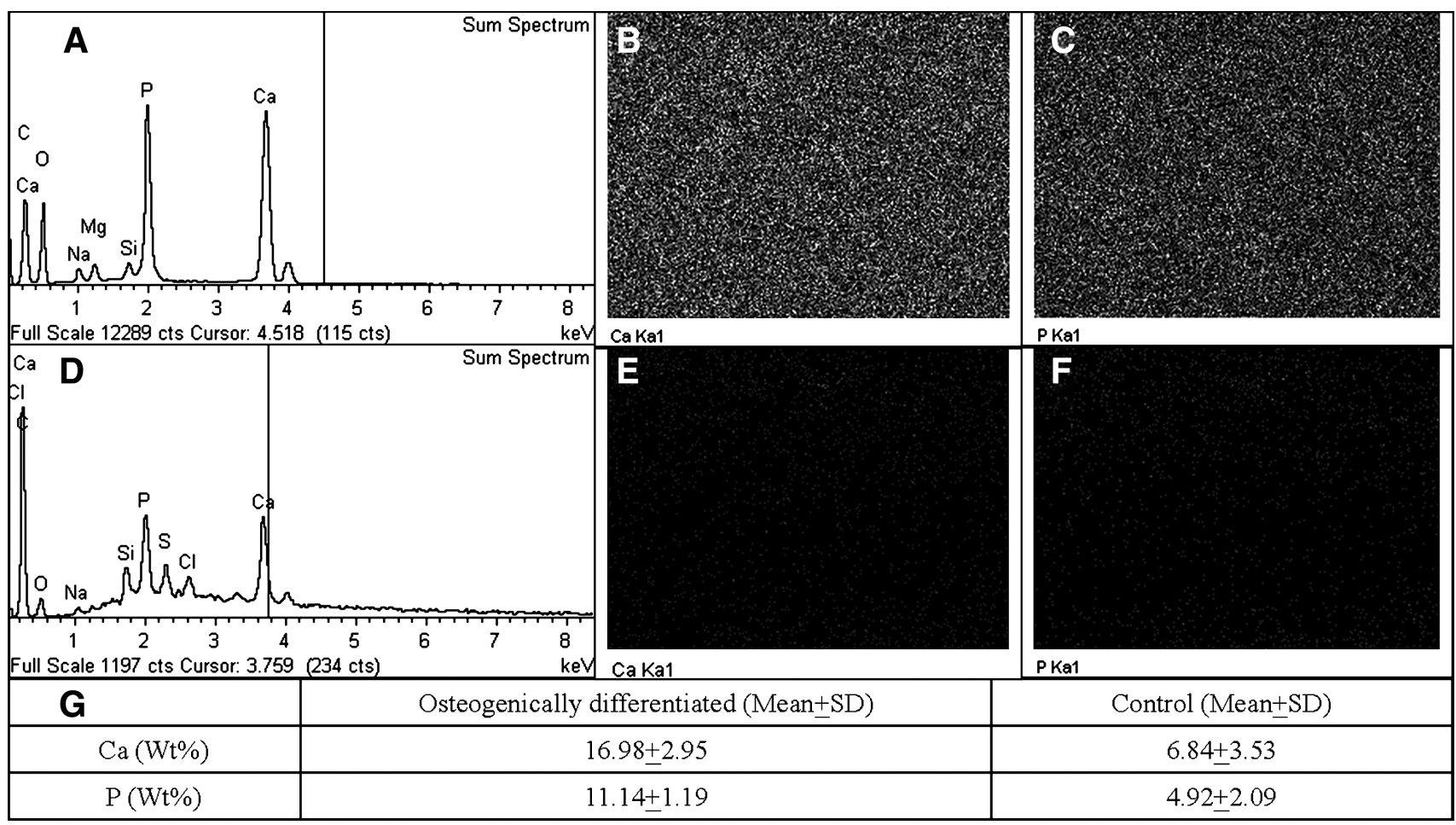

FIG. 4. Energy dispersive X-ray analysis on collagen-mMSC microspheres at 21 days postdifferentiation. Comparison between osteogenic differentiation $(\mathbf{A}-\mathbf{C})$ and control $(\mathbf{D}-\mathbf{F})$ group was shown in the energy dispersive $\mathrm{X}$-ray spectrum analysis (A, D), and mappings for calcium (B, E) and phosphorus (C, F). The mean values with standard deviations (SDs) for the relative composition of calcium and phosphorus in different groups are shown in the lower panel (G).

after complete extraction from the microspheres was conducted separately using a calcium extraction assay.

\section{Extraction of calcium deposits from the microspheres}

The dark granules or patches of the osteogenically differentiated collagen-MSC microspheres in Figure 1 are directly associated with the calcium deposition in the microsphere. This was demonstrated by the gradual removal of dark granules or patches of the microsphere during calcium extraction by trichloroacetic acid (Fig. 5). Live GFP mMSCs were present in the microsphere, and the microsphere is completely opaque and dark before extraction at time 0 . Upon addition of the acid for extraction, the dark calcium deposits were extracted in a diffusion-controlled manner over time, and complete extraction of calcium was observed within $30 \mathrm{~min}$. The microsphere becomes transparent again after complete extraction of calcium.

\section{Quantification of calcium deposition in collagen-MSC microspheres}

Figure 6 shows the dry weight and calcium contents normalized by number and dry weight of microspheres encapsulating hMSC or mMSC, in various treatment groups and at different time points. An increase in microsphere dry weight indicates increased deposition of new extracellular matrix including the calcium deposits. An increase in calcium per unit dry weight is a measure of the relative amount of calcium to the dry weight and is an indicator of the quality of bone formed. An increase in calcium per microsphere in- dicates increased osteogenic activity of the differentiating cells or increased osteogenic cells in each microsphere. In collagen-hMSC microspheres, the dry weights of microspheres between different treatment groups and at different time points were not significantly different $(p>0.05)$ (Fig. $6 \mathrm{~A})$. The percentage dry weight of calcium in differentiated microspheres ranged from $0.3 \%$ to $1.2 \%$ comparing with the minimal values in the control group, but the difference was still statistically insignificant $(p=0.09)$ (Fig. 6B). Nevertheless, the calcium per microsphere in differentiated collagenhMSC microspheres was statistically significantly different from the control group $(p=0.025)$, and the significant difference was due to the osteogenic differentiation $(p=0.008)$ but not due to the time factor $(p>0.05)$ (Fig. 6C). In microspheres encapsulating mMSCs, the dry weight of microspheres changed significantly $(p<0.001)$ and the difference was due to both time factor $(p=0.004)$ and treatment group $(p<0.001)$ (Fig. 6D). The percentage of calcium dry weight in differentiated collagen-mMSC microspheres reached $\sim 22 \%$ of the total dry weight (Fig. $6 \mathrm{E}$ ) and was comparable to that of the native bone. ${ }^{25,26}$ The change in the percentage dry weight was also statistically significant $(p=0.001)$, and the difference was due to the effect of treatment group $(p<0.001)$ but not the time factor (Fig. 6E). Finally, the calcium per microsphere in collagen-mMSC microsphere was also significantly changed $(p<0.001)$, and the difference was due to both the time factor $(p=0.005)$ and the treatment group $(p<0.001)$ (Fig. 6F). The right panel of Figure 6 shows the temporal change of the dry weight, the calcium per dry weight, and the calcium per microsphere in collagen-mMSC 

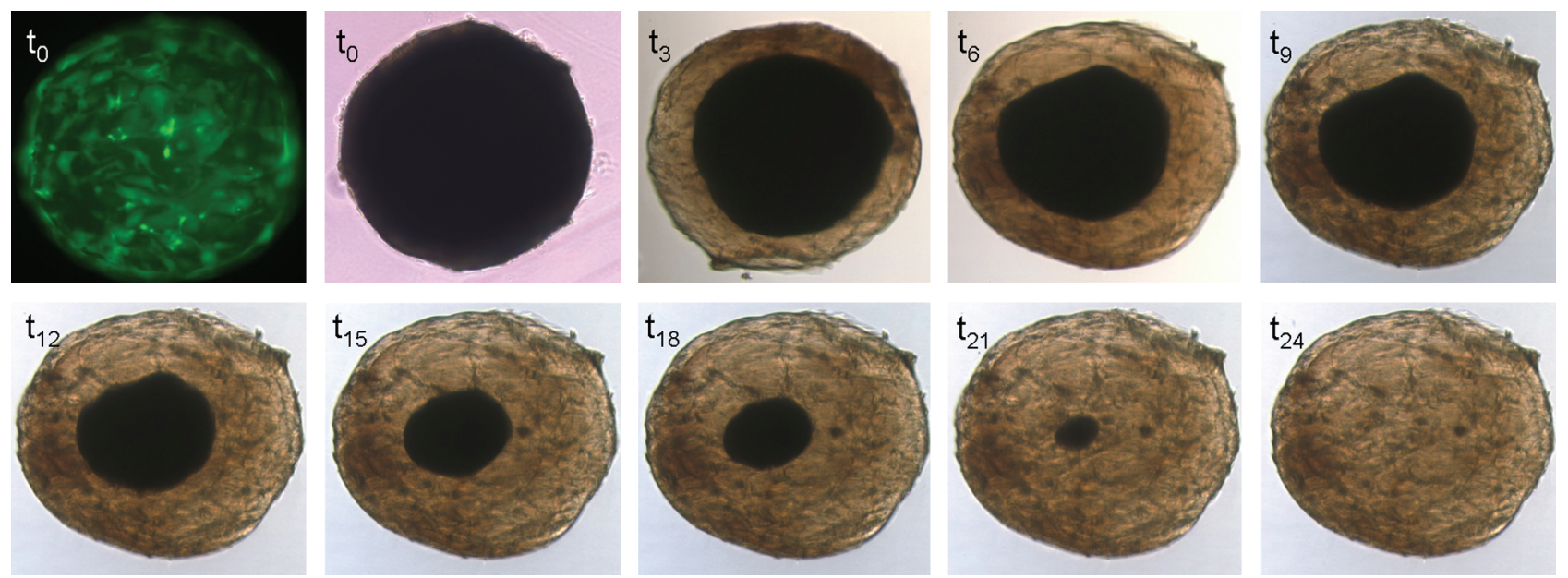

FIG. 5. Morphological change of osteogenically differentiated collagen-mMSC microspheres (21 days postdifferentiation) at different time points (min) during calcium extraction by trichloroacetic acid. Color images available online at www .liebertonline.com/ten.

A

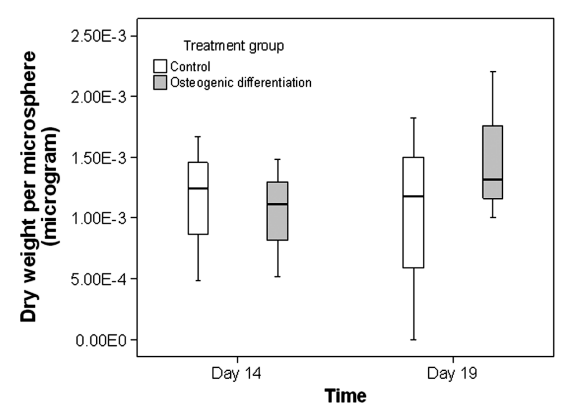

B
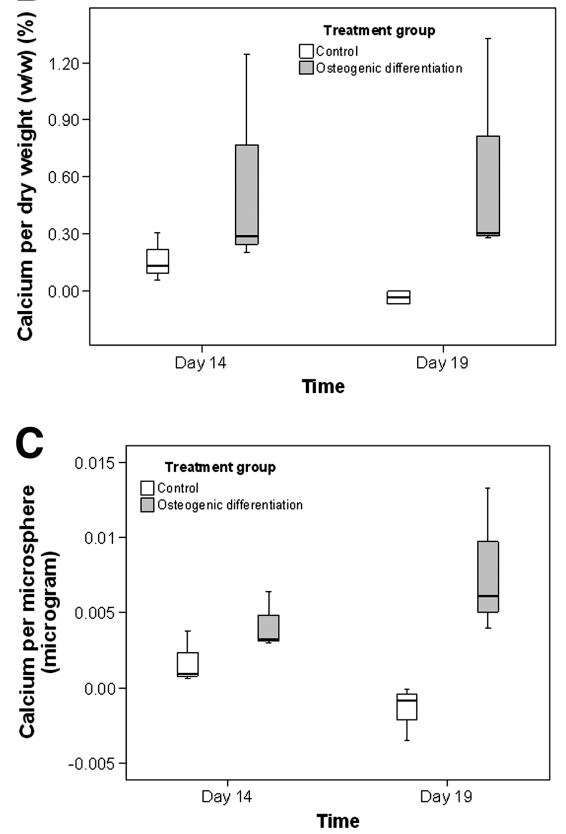

D

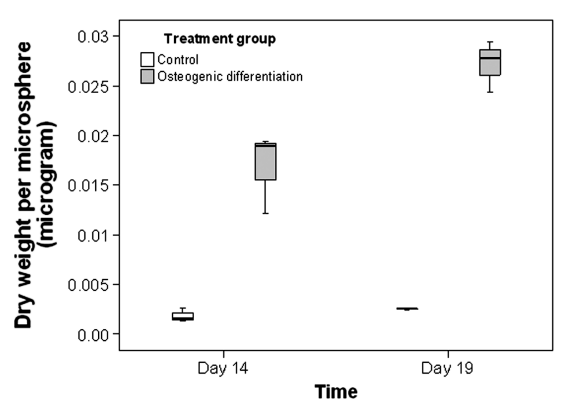

E
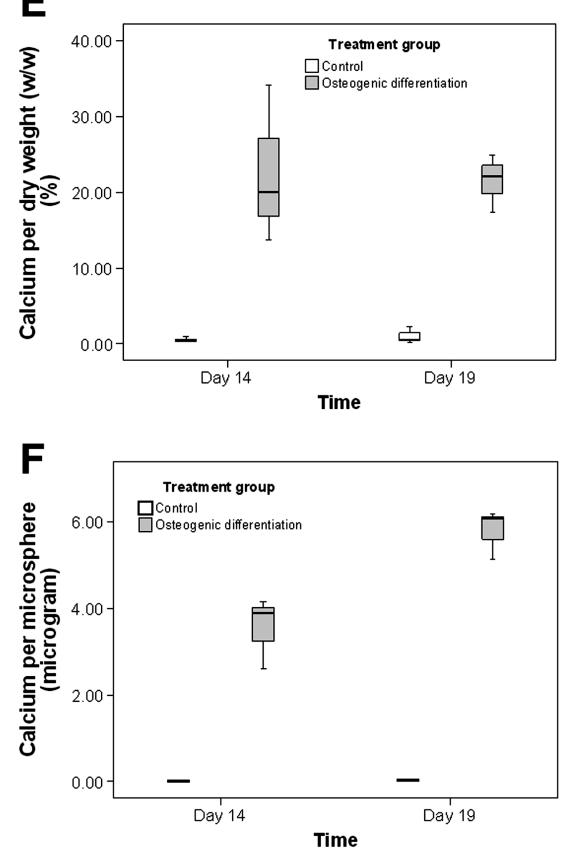

G $\quad$ mMSC
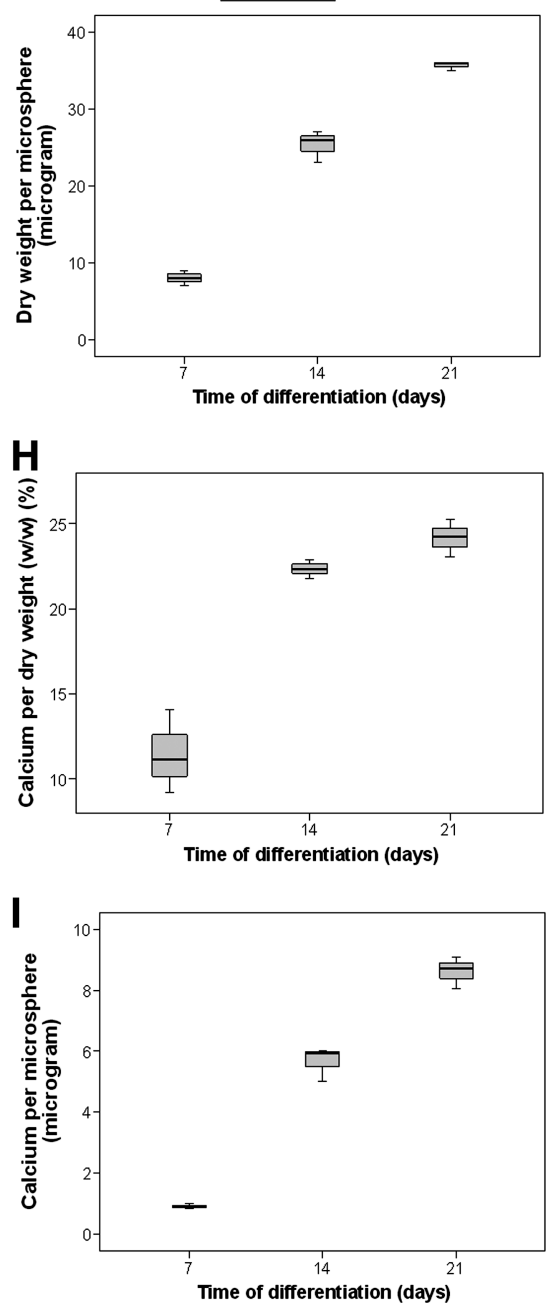

FIG. 6. Box plots showing the quantification of calcium deposits in collagen-MSC microspheres. Left panel: dry weight (A) calcium content per unit dry weight $(\mathbf{B})$ and calcium content per microsphere $(\mathbf{C})$ for collagen-hMSC microspheres in osteogenic differentiation and control groups. Middle panel: dry weight (D) calcium content per unit dry weight (E) and calcium content per microsphere $(F)$ for collagen-mMSC microspheres in osteogenic differentiation and control groups. Right panel: dry weight $(\mathbf{G})$ calcium content per unit dry weight $(\mathbf{H})$ and calcium content per microsphere (I) for collagen-mMSC microspheres at different time points during osteogenic differentiation. 
microspheres during differentiation. Temporal increase in dry weight and the normalized calcium content was statistically significant $(p<0.001)$ with positive linear trend $(p<0.001)$, demonstrating some causal relationship between the osteogenic differentiation and the calcium deposition. Post hoc Bonferroni's tests showed that values at all three different time points were significantly different from one another for dry weight $(p<0.001)$ and calcium per microsphere $(p \leq 0.001)$, while for calcium per dry weight, all but the day 14 and 21 pair showed a significant difference $(p<0.001)$.

\section{Osteoinductive activity of osteogenically differentiated collagen-mMSC microspheres}

In coculture of differentiated collagen-GFP ${ }^{+\mathrm{ve}}-\mathrm{mMSC}$ microspheres (Fig. 7B) and undifferentiated MSCs with cellcell contact (Fig. 7A-C), GFP ${ }^{-v e}$ undifferentiated MSCs were induced to differentiate toward osteogenic lineage as demonstrated by the positive ALP staining (pink) in the monolayered cultures (Fig. 7A). Positive staining was shown at sites away from the $\mathrm{GFP}^{+\mathrm{ve}}$ microspheres in the superimposed images (Fig. 7C). This result demonstrated the osteoinductivity of the differentiated collagen-mMSC microspheres in a cell-contact manner. In coculture of differentiated collagenmMSC microspheres and undifferentiated MSCs without cell-cell contact in the transwell assay (Fig. 7D-G), the undifferentiated MSCs were also induced to differentiate toward osteogenic lineage. This was demonstrated by the positive signals of the staining for ALP (pink) and calcium deposits (gray/dark) in the undifferentiated MSC monolayers (Fig. 7D, F) in the lower chamber of the transwell plate. Control group exposed to normal medium without the differentiated collagen-mMSC microspheres showed negative signal in both staining (Fig. 7E, G). This result demonstrated the osteoinductivity of the differentiated collagen-mMSC microspheres in a noncontact manner.

\section{Discussion}

This study reports the fabrication of injectable bone-like microspheres with osteoinductivity, osteoconductivity, and osteogenicity, suggesting the potential to use them in bone tissue engineering applications.

\section{Collagen microsphere with nanofibrous meshwork is an excellent osteoconductive material}

Osteoconduction is the process in which the 3D structure of a substance is conductive for the growth of newly formed bone. ${ }^{12}$ Collagen is an attractive osteoconductive material used in bone tissue engineering as it is one of the native constituents of bone. In most, if not all, previous studies using collagen for bone tissue engineering, ${ }^{27-30}$ premade porous scaffolds have been used and the MSCs were seeded in these structures after the fabrication. In the current study, MSCs were suspended in a solution of collagen before the
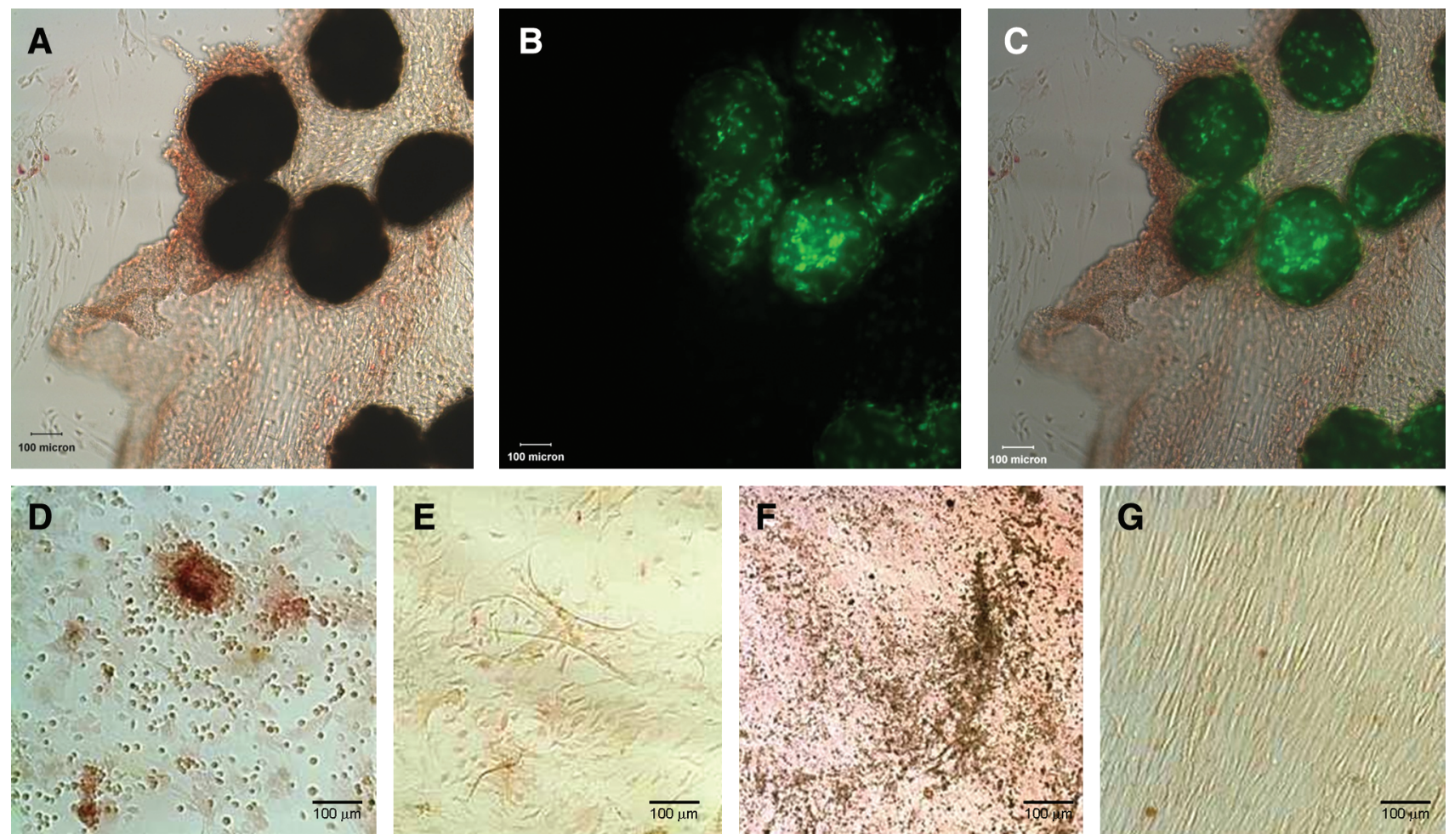

FIG. 7. Osteoinductive activity of collagen-mMSC microspheres after osteogenic differentiation. Upper panel: contact coculture of collagen-MSC microspheres (14 days postosteogenic differentiation) and undifferentiated MSCs in monolayers for 21 days after alkaline phosphatase staining under phase contrast microscopy (A), fluorescence microscopy (B), and overlay of the images (C). Lower panel: noncontact coculture of osteogenically differentiated collagen-MSC microspheres and undifferentiated MSCs in monolayers after alkaline phosphatase and von Kossa costaining in experimental (D, F) and control (E, G) groups. Magnifications: (A-C), 20×; (D-G), 10×. Color images available online at www.liebertonline.com/ten. 
reconstitution of collagen fiber is initiated. The advantages of this microencapsulation approach over the traditional cell seeding approach are (i) even distribution of the encapsulated cells throughout the microsphere, (ii) elimination of the time-consuming cell-seeding step and the inefficient cell penetration step, and (iii) optimal collagen fiber density of the meshwork entrapping the MSCs upon fiber reconstitution and matrix solidification. Nevertheless, the most important feature of the collagen microsphere contributing to osteoconduction should be its nanofibrous microstructure surrounding the encapsulated cells, ${ }^{21,22}$ as characterized by the presence of native collagen D-bands of around $67 \mathrm{~nm}$ width, ${ }^{21}$ randomly distributed fibers of $\sim 45-50 \mathrm{~nm}$ in diameter, $^{22}$ and a network of interconnected fibers with mesh size of $\sim 400 \mathrm{~nm}^{31}$ On the other hand, only macrostructures with collagen sheet-like structures and macropores of $\sim 300 \mu \mathrm{m}$ were found in freeze-dried collagen scaffolds in other groups. ${ }^{27-29}$

\section{MSC is the source for osteogenicity of the microspheres}

Osteogenicity refers to the ability to form bone via the supply of bone-forming cells. ${ }^{3}$ In fresh bone graft, bone marrow provides the source of osteogenic cells such as osteoblastic cells or undifferentiated MSCs. MSC becomes an attractive cell source for bone tissue engineering because of their multiple differentiating potential, including osteogenic lineages, ${ }^{16,17}$ in addition to other favorable characteristics such as their availability in adult individuals. In the current study, bone marrow MSCs from both human and mouse were entrapped in the osteoconductive collagen fiber meshwork and successfully differentiated toward the osteogenical lineages. Both types of differentiated MSCs deposited increasing amount of calcium phosphate within the collagen microsphere over time during differentiation. Calcium content analysis in native bones showed $\sim 23 \%$ calcium dry weight in mice ${ }^{25}$ and $19-26 \%$ in human. ${ }^{26}$ In the current study, the mean calcium dry weight of the mMSC-encapsulated microspheres after 21 days of osteogenic differentiation was $\sim 22 \%$, comparable to that of the native bone. This change in extracellular matrix of the microspheres may also lead to change in the functional properties of the structures. We observed significant improvement of mechanical properties, in terms of reduced elastic modulus, up to $\sim 1 \mathrm{GPa}$ in osteogenic differentiated collagen-mMSC microspheres (data not shown), using a microplate compression method developed in our laboratory. ${ }^{32}$ Nevertheless, whether the microplate method be used to measure the mechanical properties of hard tissues and whether the mechanical properties of the osteogenic differentiated microspheres correlate with the compositional change of the microsphere need further investigations. For those with hMSCs, although the calcium dry weight was only $0.64 \%$, constituting a small portion of the native bone, the differentiated phenotype can still be demonstrated by the significantly higher calcium content per microsphere, the positive von Kossa staining for calcium phosphate, and the presence of calcium deposits in the SEM analysis. In the same osteogenic differentiation medium using the same cell density and collagen concentration, hMSCs in collagen microspheres differentiate toward osteogenic lineage at a less mature status comparing with mMSCs based on the significantly less mineralization, suggesting that optimization on other factors affecting the differentiation potential of hMSC such as cell density, collagen concentration, and different differentiation media with supplementation of other osteoinductive signals such as BMPs may be required.

\section{Osteogenic differentiated collagen-MSC microspheres are osteoinductive}

Osteoinduction refers to a process whereby osteoinductive substances such as BMPs, ${ }^{9,10}$ calcium phosphates, ${ }^{3,33}$ and collagen $^{14}$ signal the surrounding stem cells to differentiate into bone cells rather than other lineages. ${ }^{12,24}$ In the current study, soluble osteogenic differentiation-stimulating signals have been provided to induce the osteogenic differentiation of undifferentiated MSCs encapsulated in the collagen microspheres. The encapsulated MSCs have committed to osteogenic lineages. The osteoinductive activities of these microspheres have been demonstrated through cocultures with undifferentiated MSCs. As a result, these osteoinductive microspheres entrapping committed MSCs have the potential to be transplanted or injected to bone defects to stimulate bone formation. Moreover, the osteoinductive effects of the differentiated collagen-MSC microspheres are mediated through, at least partially, substances or factors secreted by the differentiating MSCs, because osteogenic differentiation of the undifferentiated MSCs is evident even in noncontact coculture where the differentiated microspheres were cultured remotely in the upper chamber of the transwells. Nevertheless, the source of the osteoinductive substances or factors may also come from the collagen meshwork, which might soak or immobilize the factors present in the osteogenic differentiation medium during the predifferentiation of MSCs in the microspheres and then released during the coculture period. Although we only showed the osteoinductive effects in the osteogenic differentiated collagen-mMSC microspheres in the osteoinductive experiments, ongoing experiment on immunohistochemical staining of collagenhMSC microsphere with BMP-2 preliminarily demonstrated that collagen-hMSC microspheres subjected to osteogenic differentiation may also be osteoinductive due to the positive staining of BMP-2 (data not shown). Nevertheless, the exact mechanisms of the osteoinductivity of the osteogenic differentiated microspheres with both mMSC and hMSC and the identity of the secreted bioactive factors deserve further investigations.

\section{Potential applications of the injectable microspheres}

The current microencapsulation system provides injectable microspheres with osteoconductive, osteogenic, and osteoinductive activities. These microspheres can be used as stable carriers for MSC delivery, and the advantage of delivering MSCs, which have already been pushed toward the osteogenic lineage, rather than undifferentiated MSCs could be earlier bone regeneration and faster bone formation. Moreover, apart from autologous source, MSCs can be obtained from allogenic sources. Recently, allogeneic therapy using MSCs for bone regeneration has been suggested. ${ }^{34}$ The current delivery system could be used for delivering allogenic cells and as allogeneic bone grafts. Moreover, the injectable microspheres can be excellent adjunct to existing bone scaffolds to further improve the bone regeneration 
outcomes as they also provide osteogenic and osteoinductive activities.

\section{Conclusion}

This study reports fabrication of potential alternatives of fresh bone grafts using bone marrow MSC-encapsulated collagen microspheres. Both mMSCs and hMSCs have been differentiated into osteogenic lineages in 3D collagen microspheres as shown by positive expression of ALP and deposition of calcium phosphates. The collagen fiber meshwork facilitates deposition of calcium phosphates, and the calcium content has been shown significantly increased. Collagen microspheres with committed MSCs possess osteoinductive activities as demonstrated by the ability to induce undifferentiated MSCs to commit to osteogenic lineage in both contact and noncontact coculture. This study contributes to the future development of bone tissue engineering.

\section{Acknowledgments}

This work was supported by grants from Research Grant Council and Innovation and Technology Commission (ITS/133/08) of the Hong Kong Government, and seed funding from University Research Committee (10400308) and Strategic Research Theme of the University of Hong Kong (Biomedical Engineering). The authors thank Dr. Y.Y. Li for assistance with the osteoinductivity assay and Mr. K.O. Lam and Ms. Sisu Mo for assistance with the bone marrow aspiration in mice.

\section{Disclosure Statement}

No competing financial interests exist.

\section{References}

1. Cornell, C.N. Osteobiologics. Bull Hosp Jt Dis 62, 13, 2004.

2. Boyan, B.D., Ranly, D.M., McMillan, J., Sunwoo, M., Roche, K., and Schwartz, Z. Osteoinductive ability of human allograft formulations. J Periodontol 77, 1555, 2006.

3. Habibovic, P., and de Groot, K. Osteoinductive biomaterialsproperties and relevance in bone repair. J Tissue Eng Regen Med 1, 25, 2007.

4. Gazdag, A.R., Lane, J.M., Glaser, D., and Forster, R.A. Alternatives to autogenous bone graft: efficacy and indications. J Am Acad Orthop Surg 3, 1, 1995.

5. Albrektsson, T., and Johansson, C. Osteoinduction, osteoconduction and osseointegration. Eur Spine J 10 Suppl 2, S96, 2001.

6. Oklund, S.A., Prolo, D.J., Gutierrez, R.V., and King, S.E. Quantitative comparisons of healing in cranial fresh autografts, frozen autografts and processed autografts, and allografts in canine skull defects. Clin Orthop Relat Res 205, 269, 1986.

7. Anderson, M.L., Dhert, W.J., de Bruijn, J.D., Dalmeijer, R.A., Leenders, H., van Blitterswijk, C.A., and Verbout, A.J. Critical size defect in the goat's os ilium. A model to evaluate bone grafts and substitutes. Clin Orthop Relat Res 364, 231, 1999.

8. Urist, M.R. Bone: formation by autoinduction. Science 150, 893, 1965.

9. Bessa, P.C., Casal, M., and Reis, R.L. Bone morphogenetic proteins in tissue engineering: the road from the laboratory to the clinic, part I (basic concepts). J Tissue Eng Regen Med 2, 1, 2008.

10. Bessa, P.C., Casal, M., and Reis, R.L. Bone morphogenetic proteins in tissue engineering: the road from laboratory to clinic, part II (BMP delivery). J Tissue Eng Regen Med 2, 81, 2008.

11. Rabie, A.B., and Chay, S.H. Clinical applications of composite intramembranous bone grafts. Am J Orthod Dentofacial Orthop 117, 375, 2000.

12. Hak, D.J. The use of osteoconductive bone graft substitutes in orthopaedic trauma. J Am Acad Orthop Surg 15, 525, 2007.

13. LeGeros, R.Z. Properties of osteoconductive biomaterials: calcium phosphates. Clin Orthop Relat Res 395, 81, 2002.

14. Rodrigues, C.V., Serricella, P., Linhares, A.B., Guerdes, R.M., Borojevic, R., Rossi, M.A., Duarte, M.E., and Farina, M. Characterization of a bovine collagen-hydroxyapatite composite scaffold for bone tissue engineering. Biomaterials 24, 4987, 2003.

15. Rust, P.A., Kalsi, P., Briggs, T.W., Cannon, S.R., and Blunn, G.W. Will mesenchymal stem cells differentiate into osteoblasts on allograft? Clin Orthop Relat Res 457, 220, 2007.

16. Pittenger, M.F., Mackay, A.M., Beck, S.C., Jaiswal, R.K., Douglas, R., Mosca, J.D., Moorman, M.A., Simonetti, D.W., Craig, S., and Marshak, D.R. Multilineage potential of adult human mesenchymal stem cells. Science 284, 143, 1999.

17. Heino, T.J., and Hentunen, T.A. Differentiation of osteoblasts and osteocytes from mesenchymal stem cells. Curr Stem Cell Res Ther 3, 131, 2008.

18. Siddappa, R., Fernandes, H., Liu, J., van Blitterswijk, C., and de Boer, J. The response of human mesenchymal stem cells to osteogenic signals and its impact on bone tissue engineering. Curr Stem Cell Res Ther 2, 209, 2007.

19. Horwitz, E.M., Prockop, D.J., Fitzpatrick, L.A., Koo, W.W., Gordon, P.L., Neel, M., Sussman, M., Orchard, P., Marx, J.C., Pyeritz, R.E., and Brenner, M.K. Transplantability and therapeutic effects of bone marrow-derived mesenchymal cells in children with osteogenesis imperfecta. Nat Med 5, 309, 1999.

20. Chan, B.P., Chan G.C.F., Wong, H.L., Cheung, P.T., Cheah, K., and Chan, D. Cell-Matrix Microsphere, Associated Products, Methods for Preparation and Applications. US Regular Patent Application No. 11/750,863 (filed on 18 May 2007) (Publication date 7 Feb 2008).

21. Chan, B.P., Hui, T.Y., Yeung, C.W., Li, J., Mo, I., and Chan, G.C. Self-assembled collagen-human mesenchymal stem cell microspheres for regenerative medicine. Biomaterials 28, 4652, 2007.

22. Hui, T.Y., Cheung, K.M., Cheung, W.L., Chan, D., and Chan, B.P. In vitro chondrogenic differentiation of human mesenchymal stem cells in collagen microspheres: influence of cell seeding density and collagen concentration. Biomaterials 29, 3201, 2008.

23. Li, J., Law, H.K., Lau, Y.L., and Chan, G.C. Differential damage and recovery of human mesenchymal stem cells after exposure to chemotherapeutic agents. Br J Haematol 127, 326, 2004.

24. Friedenstein, A.Y. Induction of bone tissue by transitional epithelium. Clin Orthop Relat Res 59, 21, 1968.

25. Whelton, B.D., Bhattacharyya, M.H., Peterson, D.P., Moretti, E.S., Toomey, J.M., and Williams, L.L. Skeletal changes in multiparous and nulliparous mice fed a nutrient-deficient diet containing cadmium. Toxicology 91, 235, 1994. 
26. Biltz, R.M., and Pellegrino, E.D. The chemical anatomy of bone. I. A comparative study of bone composition in sixteen vertebrates. J Bone Jt Surg Am 51, 456, 1969.

27. George, J., Kuboki, Y., and Miyata, T. Differentiation of mesenchymal stem cells into osteoblasts on honeycomb collagen scaffolds. Biotechnol Bioeng 95, 404, 2006.

28. Farrell, E., Byrne, E.M., Fischer, J., O'Brien, F.J., O'Connell, B.C., Prendergast, P.J., and Campbell, V.A. A comparison of the osteogenic potential of adult rat mesenchymal stem cells cultured in 2-D and on 3-D collagen glycosaminoglycan scaffolds. Technol Health Care 15, 19, 2007.

29. Donzelli, E., Salvadè, A., Mimo, P., Viganò, M., Morrone, M., Papagna, R., Carini, F., Zaopo, A., Miloso, M., Baldoni, M., and Tredici, G. Mesenchymal stem cells cultured on a collagen scaffold: in vitro osteogenic differentiation. Arch Oral Biol 52, 64, 2007.

30. Shih, Y.R., Chen, C.N., Tsai, S.W., Wang, Y.J., and Lee, O.K. Growth of mesenchymal stem cells on electrospun type I collagen nanofibers. Stem Cells 24, 2391, 2006.

31. Chan, O.C.M., So, K.F., and Chan, B.P. Fabrication of nanofibrous collagen microspheres for protein delivery and effects of photochemical crosslinking on release kinetics. J Control Release 129, 135, 2008.

32. Chan, B.P., Li, C.H., Au-yeung, K.L., Sze, K.Y., and Ngan, A.H.W. A microplate compression method for elastic mod- ulus measurement of soft and viscoelastic collagen microspheres. Ann Biomed Eng 36, 1254, 2008.

33. Müller, P., Bulnheim, U., Diener, A., Lüthen, F., Teller, M., Klinkenberg, E.D., Neumann, H.G., Nebe, B., Liebold, A., Steinhoff, G., and Rychly, J. Calcium phosphate surfaces promote osteogenic differentiation of mesenchymal stem cells. J Cell Mol Med 12, 281, 2008.

34. Arinzeh, T.L. Mesenchymal stem cells for bone repair: preclinical studies and potential orthopedic applications. Foot Ankle Clin 10, 651, 2005.

Address correspondence to: Barbara Pui Chan, Ph.D. Medical Engineering Program Department of Mechanical Engineering

The University of Hong Kong Room 711, Haking Wong Building Pokfulam Road

Hong Kong Special Administrative Region 852

China

E-mail: bpchan@hkucc.hku.hk

Received: December 31, 2008

Accepted: June 4, 2009

Online Publication Date: July 8, 2009 
